\title{
BRAF fusions in pediatric histiocytic neoplasms define distinct therapeutic responsiveness to RAF paradox breakers
}

\author{
Payal Jain ${ }^{1}$, Lea Surrey ${ }^{2}$, Joshua Straka ${ }^{3}$, Richard Womer ${ }^{2}$, Marilyn $\mathrm{Li}^{3}$, Phillip Storm ${ }^{4}$, \\ Angela Waanders ${ }^{5}$, Michael Hogarty ${ }^{2}$, Adam Resnick ${ }^{2}$, and Jennifer Picarsic ${ }^{6}$ \\ ${ }^{1}$ The Children's Hospital of Philadelphia Research Institute \\ ${ }^{2}$ Children's Hospital of Philadelphia \\ ${ }^{3}$ The Children's Hospital of Philadelphia \\ ${ }^{4}$ University of Pennsylvania \\ ${ }^{5}$ Ann and Robert H Lurie Children's Hospital of Chicago \\ ${ }^{6}$ Cincinnati Children's Hospital Medical Center
}

December 14, 2020

\begin{abstract}
Pediatric histiocytic neoplasms are hematopoietic disorders frequently driven by the BRAF-V600E mutation. Here we identified two BRAF gene fusions (novel MTAP-BRAF and MS4A6A-BRAF) in two aggressive histiocytic neoplasms. In contrast to previously described BRAF fusions, MTAP-BRAF and MS4A6A-BRAF do not respond to the paradox breaker RAF inhibitor (RAFi) PLX8394 due to stable fusion dimerization mediated by the N-terminal fusion partners. This highlights a significant and clinically relevant shift from the current dogma that BRAF-fusions respond similarly to BRAF-inhibitors. As an alternative, we show suppression of fusion-driven oncogenic growth with the pan-RAFi LY3009120 and MEK inhibition.
\end{abstract}

\section{Introduction}

Histiocytic neoplasms are a diverse group of clonal hematopoietic disorders that are driven by mutations activating the mitogen-activated protein kinase (MAPK) and phosphoinositide 3-kinases (PI3K) pathways ${ }^{1-3}$. While $B R A F-\mathrm{V} 600 \mathrm{E}$ is the most common alteration in histiocytic neoplasms, multiple alternate pathway activating mechanisms have been described, including MAP2K1 ,ARAF , PIK3CA, NRAS , and KRAS mutations as well as BRAF, ALK, and NTRK1 fusions ${ }^{1-3}$. BRAF-fusions previously reported in cases of histiocytic neoplasms ${ }^{1,4-7}$ were found to contain the N-terminal region of another gene (often of unclear significance) joined to the BRAF kinase domain (including exons 9-18 or 11-18), resulting in the loss of the BRAF N-terminal regulatory RAS-binding regions (exons 1-8). Despite the prevalence of BRAF-alterations in histiocytic tumors, to date there have been no detailed molecular investigations comparing BRAF-fusions found in distinct sub-types of histiocytic neoplasms and only one study explored responsiveness of BRAFfusions to single-agent RAF-therapies ${ }^{4}$. To address this, we present two pediatric histiocytosis cases, with distinct pathologic features and clinical behavior, each harboring a BRAF-fusion identified by next-generation sequencing (Supplemental Methods and Table S1) and study their in vitro responsiveness to RAF-targeted inhibitors.

\section{Results}

Case 1, a 16 year-old female with a $2.5 \mathrm{~cm}$ rapidly growing subcutaneous thigh mass was diagnosed with a malignant histiocytic neoplasm ("M group"), with high-grade morphologic features and a phenotype spanning histiocytic sarcoma (CD163/CD14/CD68+) and Langerhans cell sarcoma (CD1a/Langerin/S100) 
with a modestly elevated Ki-67 proliferation index (up to 20\%) (Figure 1A-F). Targeted RNA-sequencing identified a MTAP-BRAF fusion transcript. Resection margins were negative. The patient is diseasefree three years post-resection. Case 2, a 12-year-old female with a $5.3 \mathrm{~cm}$ rapidly enlarging heel mass invading the calcaneus was originally diagnosed with a juvenile xanthogranuloma (JXG) family lesion (CD163/CD68/CD14/fascin/Factor XIIIa+) (Figure 1G-M). Despite low-grade morphologic features and lack of cytologic atypia or increased mitotic rate by H\&E stain, a high proliferation rate (up to $40 \%$ ) was noted by Ki-67 proliferation index stain. Targeted RNA-seq identified a MS4A6A-BRAF fusion transcript. During staging, the patient was found to have PET-avid dissemination to lymph nodes and lung (Figure 1N-P). While the initial morphologic features were consistent with a low-grade histiocytic lesion of JXG phenotype, the integration of the high Ki-67 proliferation index and aggressive clinical behavior with lymphatic/metastatic-like spread, along with a novel molecular BRAF-fusion, at the time of diagnosis, suggested an atypical JXG family neoplasm with uncertain biological behavior. The patient was treated with 12 cycles of clofarabine with clinical remission of metastatic sites and near clinical remission at primary site now 18 months off therapy.

MTAP-BRAF and MS4A6A-BRAF fusions are predicted to contain all functional domains of MTAP and MS4A6A, respectively, along with the BRAF kinase domain but no N-terminal regulatory, RAS-binding domain (Figure 1Q). For molecular and therapeutic characterization, MTAP-BRAFand MS4A6A-BRAF were cloned and stably expressed in a heterologous cell model since patient-derived cell lines were lacking. The NIH/3T3 cells model system was utilized for its ability to reliably discern oncogenic fusion profiles ${ }^{9-11}$. In soft agar assays, both MTAP-BRAF and MS4A6A-BRAF expressing NIH3T3 showed a significant increase in colony count over control $(\mathrm{p}<0.0001$, Figure $1 \mathrm{R})$. Next, we tested activation of downstream MAPK and PI3K/mTOR pathways. Upon serum starvation, we observed elevated levels of phosphorylated-ERK and -S6 in both BRAF- fusion expressing cells compared to controls, indicating aberrant activation of the MAPK and PI3K/mTOR pathways, respectively (Figure 1S). Slightly higher PI3K/mTOR pathway activation levels in MTAP-BRAF versus MS4A6A-BRAF cells are partly explained by higher MTAP-BRAF protein expression (Figure 1S, Myc-tag blot).

A single report on BRAF- fusions in $\mathrm{LCH}^{4}$ has shown unresponsiveness to first generation specific BRAFV600E inhibitors (RAFi) such as vemurafenib, but observed suppression by second-generation RAFi, PLX8394, and downstream MEK inhibition, similar to other pediatric glioma studies on BRAF-fusions ${ }^{9,11}$. Herein, we evaluated the responsiveness of MTAP-BRAF and MS4A6A-BRAF to such targeted inhibitors. Upon targeting the NIH3T3 models with first-generation RAFi PLX4720, as expected, no suppression of BRAF-fusion driven signaling or growth was observed (Supplemental Figure S1A). Interestingly, secondgeneration RAFi PLX8394 also showed no suppression in MTAP- or MS4A6A-BRAF driven soft agar growth despite targeting MAPK/PI3K signaling (Figure 2A-B). This is in contrast to PLX8394-mediated suppression of BRAF-fusion driven growth in the previously described $\mathrm{LCH}^{4}$ and other cancers, such as the KIAA1549-BRAF fusion in pediatric glioma ${ }^{9-11}$. PLX8394 suppressed FAM131B-BRAF (a pediatric glioma derived fusion ${ }^{12,13}$ ) and BRAF-V600E driven growth and signaling as well as actively disrupted FAM131B-BRAF dimers (Supplemental Figures S1B-D), highlighting therapeutic differences between MTAP-/MS4A6A-BRAF, BRAF-V600E and other BRAF-fusions.

BRAF- fusions function as active homo- and heterodimers (with wild-type BRAF) to mediate cell signaling ${ }^{9,11}$. We found that MTAP- and MS4A6A-BRAF also mediate such protein-protein interactions in co-immunoprecipitation assays (Figure 2C-D, DMSO lanes). PLX8394 blocks BRAF kinase activity via disrupting BRAF dimerization ${ }^{14}$ but we observed no disruption of MTAP- and MS4A6A-BRAF fusion dimerization with PLX8394 (Figure 2C-D, PLX8394 lanes), thereby providing a plausible explanation for PLX8394 unresponsiveness in soft agar assays though MAPK/PI3K signaling remains discordantly suppressed by some unknown mechanism. This distinct unresponsiveness to pan-RAFi represents a significant departure from the current view that $B R A F$-fusions and other $B R A F$ mutations should respond to second-generation RAFi such as PLX8394 ${ }^{9,15}$. We found that this difference arises due to the contribution of N-terminal partners, MTAP (exons 1-7) and MS4A6A (exons 1-6), to respective fusion dimerization that is unaffected by PLX8394 (Figure 2C-D, lanes 3,7). Similar role of N-terminal partner accounts for differential response of CRAF- fu- 
sions to PLX8394 ${ }^{10}$. Furthermore, we observed that Trunc-MTAP (exons 1-7) competitively substituted MTAP-BRAF homo-dimerization in a dose-dependent manner, suggesting preferential and potent protein interactions mediated by the N-terminal partner in these histiocytic-specific BRAF-fusions (Figure 2E).

To target dimerization-dependent oncogenicity of MTAP- and MS4A6A-BRAF via a different mechanism, we tested LY3009120, a pan-RAF dimer inhibitor that binds and stabilizes the BRAF dimer in an inactive conformation ${ }^{16}$. LY3009120 showed robust suppression of both fusion-mediated signaling and colony transformation (Figure 2B) while stabilizing the MTAP- or MS4A6A-BRAF in inactive conformation (Figure 2C-D, respectively, lanes 9-11). We also tested the effect of FDA-approved MEK inhibitors (MEKi) ${ }^{17}$, selumetinib and trametinib. We observed dose-dependent decrease in phospho-ERK and growth with trametinib (Figure 2F) and selumetinib (Supplemental Figure S2) in both BRAF-fusion models suggesting downstream MEKi as a therapeutic alternative to RAFi.

\section{Discussion}

Our findings highlight the unique and differential biology hijacked by select BRAF fusions, which impacts RAFi responsiveness. Though functional data predicts similar effects for these two novel fusions, each of these histiocytic neoplasms had a discordant clinical to pathologic behavior. Typical JXG family lesions often show indolent behavior in pediatric patients ${ }^{19}$. Even in rare systemic presentations, they do not typically feature a lymphatic-type dissemination, as demonstrated in case 2. Furthermore, Ki-67 proliferation index in JXG lesions is typically less than $20 \%$, and more often no higher than $10 \%$ (unpublished data, JP). The focally high proliferation rate (up to 40\%) in this MS4A6A-BRAF JXG family lesion appeared to correlate with its aggressive clinical presentation, despite its seemingly low-grade morphology. On the contrary, malignant histiocytic neoplasms lesions typically have aggressive clinical behavior ${ }^{18}$, unlike case 1 , which after surgical excision had an indolent course.

The MTAP-BRAF malignant histiocytic neoplasm had only modestly elevated proliferation rate (up to $20 \%$ ), which is lower than most malignant histiocytic neoplasms (often $>30 \%)^{20}$. Thus, the lower Ki-67 proliferation rate, also appeared to correlate with its indolent behavior, despite its high-grade morphology. Both of these unusual, divergent phenotypes further emphasize that in histiocytic neoplasms, the integration of histopathologic, molecular, and clinical/radiographic data are required to obtain a comprehensive assessment of clinical aggressiveness and nominate rational treatment options. A detailed mechanistic classification of BRAF fusions that predict responsiveness to targeted agents is warranted.

\section{Acknowledgments:}

The authors would like to thank Dr. Ronald Jaffe for second review of the cases and appraisal of the original manuscript.

This work was supported by the Children's Brain Tumor Tissue Consortium funding sources (P.J., A.J.W., P.B.J., and A.C.R).

\section{Authorship Contribution:}

J.P., L.F.S, P.J. conceived the study; J.P., L.F.S and P.R. generated, analyzed and interpreted the pathology data; P.J. and J.S. performed molecular experiments as well as analyzed and interpreted the results; P.J., L.F.S, J.S. and J.P. wrote the manuscript and compiled the figures; P.J., L.F.S, J.S., P.R., R.W., M.L., P.B.S., A.J.W., M.D.H., A.C.R and J.P. edited and reviewed the manuscript.

Conflict-of-interest disclosure: The authors declare no competing financial interests.

Correspondence: Jennifer Picarsic, Department of Pathology, University of Pittsburgh School of Medicine Pittsburgh, PA USA, e-mail:jenpicarsic@gmail.com

References:

1. Diamond EL, Durham BH, Haroche J, et al. Diverse and Targetable Kinase Alterations Drive Histiocytic Neoplasms. Cancer Discov . 2016;6(2):154-165. 
2. Durham BH, Diamond EL, Abdel-Wahab O. Histiocytic neoplasms in the era of personalized genomic medicine. Curr Opin Hematol . 2016;23(4):416-425.

3. Ozkaya N, Dogan A, Abdel-Wahab O. Identification and Targeting of Kinase Alterations in Histiocytic Neoplasms. Hematol Oncol Clin North Am . 2017;31(4):705-719.

4. Chakraborty R, Burke TM, Hampton OA, et al. Alternative genetic mechanisms of BRAF activation in Langerhans cell histiocytosis. Blood . 2016;128(21):2533-2537.

5. Zarnegar S, Durham BH, Khattar P, et al. Novel activating BRAF fusion identifies a recurrent alternative mechanism for ERK activation in pediatric Langerhans cell histiocytosis. Pediatr Blood Cancer . 2018;65(1).

6. Lee LH, Gasilina A, Roychoudhury J, et al. Real-time genomic profiling of histiocytoses identifies earlykinase domain BRAF alterations while improving treatment outcomes. JCI Insight . 2017;2(3):e89473.

7. Durham BH, Lopez Rodrigo E, Picarsic J, et al. Activating mutations in CSF1R and additional receptor tyrosine kinases in histiocytic neoplasms. Nat Med . 2019;25(12):1839-1842.

8. Emile JF, Abla O, Fraitag S, et al. Revised classification of histiocytoses and neoplasms of the macrophagedendritic cell lineages. Blood . 2016;127(22):2672-2681.

9. Sievert AJ, Lang SS, Boucher KL, et al. Paradoxical activation and RAF inhibitor resistance of BRAF protein kinase fusions characterizing pediatric astrocytomas. Proc Natl Acad Sci U S A . 2013;110(15):59575962 .

10. Jain P, Fierst TM, Han HJ, et al. CRAF gene fusions in pediatric low-grade gliomas define a distinct drug response based on dimerization profiles. Oncogene . 2017;36(45):6348-6358.

11. Jain P, Silva A, Han HJ, et al. Overcoming resistance to single-agent therapy for oncogenic BRAF gene fusions via combinatorial targeting of MAPK and PI3K/mTOR signaling pathways. Oncotarget . 2017;8(49):84697-84713.

12. Cin H, Meyer C, Herr R, et al. Oncogenic FAM131B-BRAF fusion resulting from 7q34 deletion comprises an alternative mechanism of MAPK pathway activation in pilocytic astrocytoma. Acta Neuropathol . 2011;121(6):763-774.

13. Roth JJ, Santi M, Pollock AN, et al. Chromosome band 7q34 deletions resulting in KIAA1549-BRAF and FAM131B-BRAF fusions in pediatric low-grade Gliomas. Brain Pathol . 2015;25(2):182-192.

14. Zhang C, Spevak W, Zhang Y, et al. RAF inhibitors that evade paradoxical MAPK pathway activation. Nature . 2015;526(7574):583-586.

15. Okimoto RA, Lin L, Olivas V, et al. Preclinical efficacy of a RAF inhibitor that evades paradoxical MAPK pathway activation in protein kinase BRAF-mutant lung cancer. Proc Natl Acad Sci US A. 2016;113(47):13456-13461.

16. Peng SB, Henry JR, Kaufman MD, et al. Inhibition of RAF Isoforms and Active Dimers by LY3009120 Leads to Anti-tumor Activities in RAS or BRAF Mutant Cancers. Cancer Cell . 2015;28(3):384-398.

17. Gilmartin AG, Bleam MR, Groy A, et al. GSK1120212 (JTP-74057) is an inhibitor of MEK activity and activation with favorable pharmacokinetic properties for sustained in vivo pathway inhibition. Clin Cancer Res . 2011;17(5):989-1000.

18. Jacobsen E, Abla O, Visser J. Malignant Histiocytoses. In: Abla O, Janka G, eds. Histiocytic Disorders. Cham: Springer International Publishing; 2018:361-381.

19. Ceppi F, Abla O. Juvenile Xanthogranuloma and Related Non-LCH Disorders. In: Abla O, Janka G, eds. Histiocytic Disorders. Cham: Springer International Publishing; 2018:293-311. 
20. Picarsic J, Jaffe R. Pathology of Histiocytic Disorders and Neoplasms and Related Disorders. In: Abla O, Janka G, eds. Histiocytic Disorders. Cham: Springer International Publishing; 2018:3-50.

Figure Legends:

Figure 1. Novel BRAF-fusions in histiocytic neoplasms mediate oncogenicity via activation of MAPK/PI3K/mTOR pathway: Malignant histiocytic neoplasm with histiocytic and Langerhans cell sarcoma phenotypes with novel $M T A P-B R A F$ fusion, and atypical juvenile xanthogranuloma family lesion with novel $M S 4 A 6 A-B R A F$ fusion.

A-F. Case 1 Malignant histiocytic neoplasm with large, pleomorphic cells (A-B) and areas of necrosis $\left(^{*}\right)$. Immunohistochemistry with CD163 (C), CD1a (D) and Langerin (E) in a subset of lesional cells. Ki-67 proliferation index (F) was elevated up to $20 \%$, including atypical large cells (F). (Original magnification A. 200x, B, 4000x, C-E. 1000x, F. 200x). BRAF VE1 immunostain was negative (not shown).G-M. Case 2 atypical juvenile xanthogranuloma (JXG) family neoplasm with bland histiocytes (G-H) and a rare mitosis $(\mathrm{H}$, center). Immunohistochemistry with Factor XIIIa (I) was strongly and diffusely positive. The Ki67 proliferation index was variable, as high as $40 \%$ (J-K) in one core biopsy and as low as $10 \%$ in other core (L-M) taken at the same time and accounting for inflammation, which was low in both core biopsies. (Original magnification: G. 100x, H. 1000x, I. 200x, J. 100x, K. 1000x, L. 100x, M. 1000x). The BRAF VE1 immunostain was negative (not shown). N-P. Case 2 with JXG: Imaging at diagnosis revealed a crescentic enhancing soft tissue mass by magnetic resonance imaging wrapping around the calcaneus, deep to the Achilles tendon (N, arrows) and positron emission tomography (PET) scanning revealed abnormal signal in the ankle (primary), knee, inguinal region and chest $(\mathrm{O})$. Following 9 of 12 cycles of clofarabine, PET scan revealed resolution of disseminated disease and shrinkage of the primary ankle tumor. Q . Structure of novel BRAF-fusions in histiocytic neoplasms. MTAP-BRAF: MTAP exons 1-7 encode phosphate binding sites, trimerization site at Trp189 residue, and substrate binding site, and BRAF exons 9-18 encode the tyrosine kinase domain. MS4A6A-BRAF: MS4A6A exons 1-6 encode 4 transmembrane helical regions, and BRAF exons 11-18 encode the tyrosine kinase domain. R. Soft agar assay using NIH3T3 cells stably expressing BRAF-fusions. Error bars represent SEM, $\mathrm{n}=5,{ }^{* * *}$ p-value $<0.001$ compared with control conditions.S. Western blot analysis of MAPK and PI3K/mTOR pathway proteins in NIH3T3 cells stably expressing BRAF-fusions. 'p-' and 't-' represent phosphorylated and total versions of protein, respectively.

Figure 2. MTAP-BRAF and MS4A6A-BRAF fusions are not suppressed by second generation RAF inhibitors but demonstrate sensitivity to LY3009120 and MEK inhibitors.

A. Western blot analysis (left) and soft agar colony counts (right) showing the effect of second generation RAFi, PLX8394, on NIH3T3 cells expressing MTAP-BRAF and MS4A6A-BRAF respectively. B. Western blot analysis (left) and soft agar colony counts (right) showing the effect of pan-RAF-dimer inhibitor, LY3009120, on NIH3T3 cells expressing MTAP-BRAF and MS4A6A-BRAF respectively. C. Co-immunoprecipitation (co-IP) assay assessing homo-dimerization of MTAP-BRAF as well as heterodimerization with wild-type BRAF and Trunc-MTAP in HEK293 cells under control, PLX8394, and LY3009120 treated conditions. D.Co-immunoprecipitation assay assessing homo-dimerization of MS4A6ABRAF as well as hetero-dimerization with wild-type BRAF and Trunc-MS4A6A in HEK293 cells under control, PLX8394, and LY3009120 treated conditions.E. Competition co-IP assay assessing preferential interaction of Trunc. MTAP with MTAP-BRAF fusion versus homo-dimerization. Increasing doses of tetracycline $(0,0.1,0.5,1 \mathrm{ug} / \mathrm{ml})$ used to regulate protein level of His-tagged Trunc-MTAP. F. Western blot analysis (left) and soft agar colony counts (right) showing the effect of MEK inhibitor, trametinib, on NIH3T3 cells expressing MTAP-BRAF and MS4A6A-BRAF respectively. Error bars represent SEM, $n=3$. No value on bar represents NS (non-significant), ${ }^{*}$-value $<0.05,{ }^{*}$ p-value $<0.01,{ }^{* * *}$ p-value $<0.001$ compared with control conditions. 'p-' and 't-'represent phosphorylated and total versions of protein, respectively. 
Figure 1:
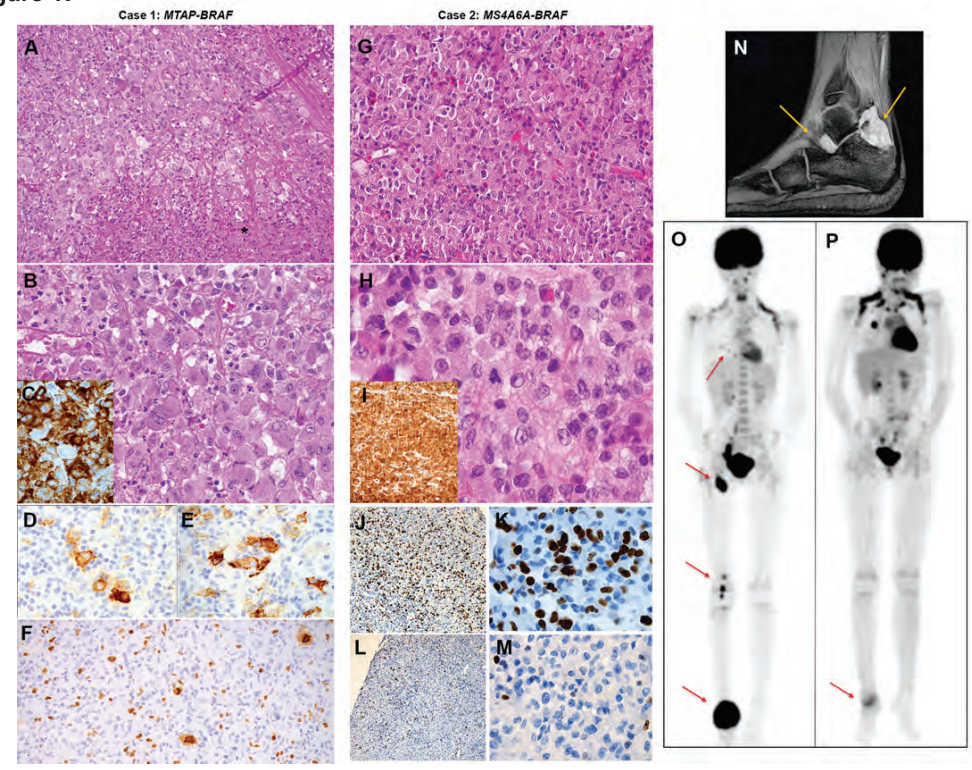

Q.

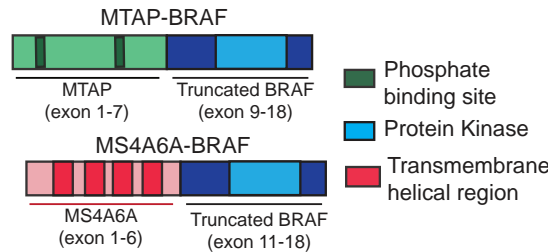

R.

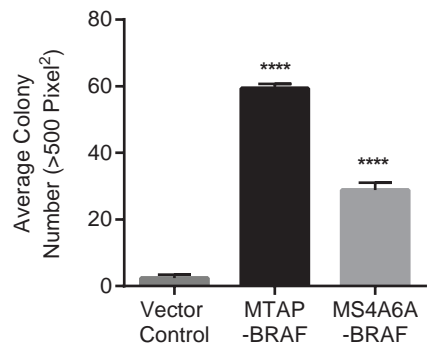

S.

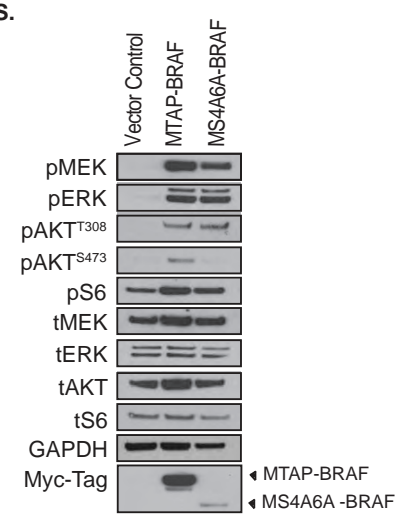


Figure 2

A.
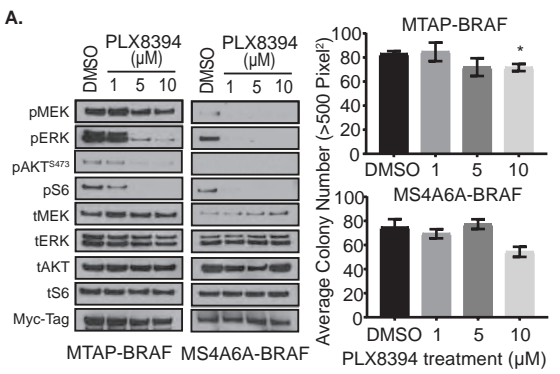

B.

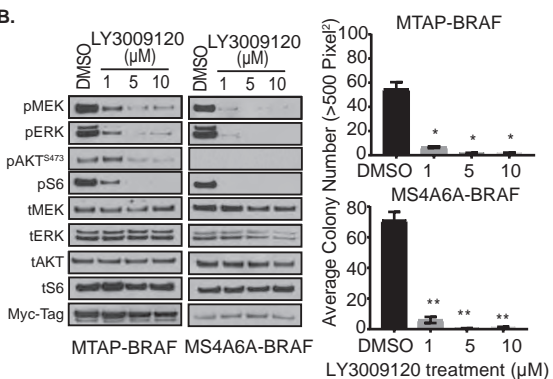

c.

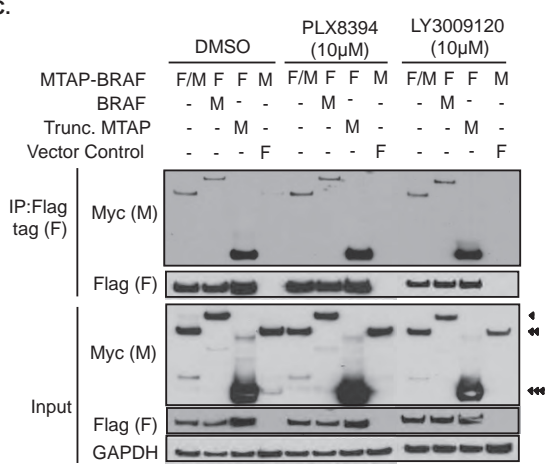

E.

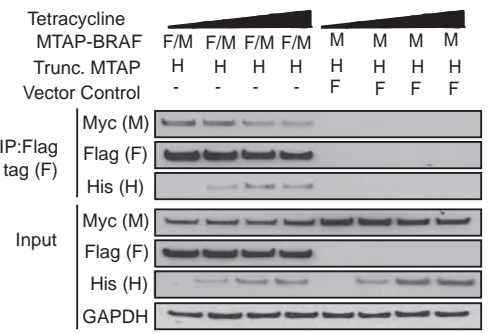

D.

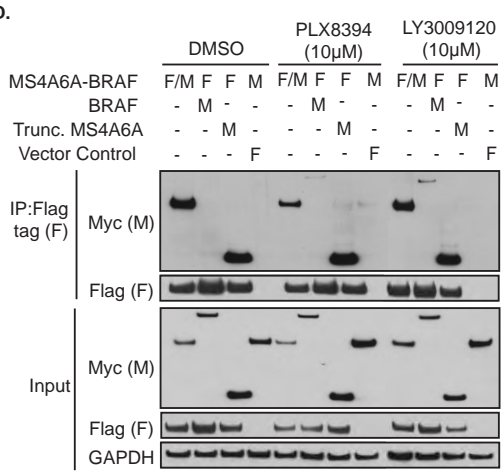

F.

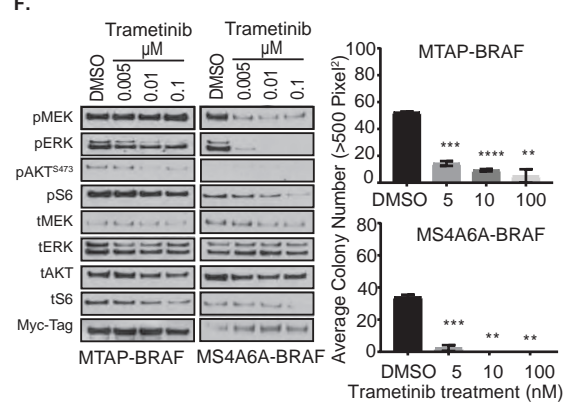

